\title{
Timed fibrin digestion: A simplified technique for the measurement of the fibrinolytic activity of the blood
}

\author{
J. A. HICKMAN AND I. C. GORDON-SMITH \\ From the Department of Surgical Studies, The Middlesex Hospital, London
}

SYNOPSIS When the rate of fibrinolysis in vitro was measured by an isotopic technique, it was found that the amount of fibrin digested after incubation for three hours correlated closely with the overall rate of the digestion process. It is suggested that, using an isotopic technique, estimation of fibrin digestion after three hours' incubation provides a useful method for measuring the fibrinolytic activity of blood. A recommended technique is described and its advantages are discussed briefly.

By employing ${ }^{125}$ I-labelled fibrinogen in vivo, the fibrinolytic activity of blood can be measured in vitro by observing the rate of release of radioactivity from blood samples diluted in buffer and clotted with thrombin (Hickman, 1971). A description of the technique is given in Figure 1. Studies which are given below show that the amount of fibrin digested after incubation for three hours correlates closely with the overall rate of the reaction. A simplified technique is therefore described for the estimation of the three-hour fibrin digestion, and the experimental justification for its application is given.

\section{Materials and Methods}

The isotopic method, the method for estimation of plasma fibrinogen concentration, and the reagents used were as described previously (Hickman, 1971), except that the measurement of fibrin digestion at three hours was performed in triplicate.

Ninety-two studies were included in the analysis. For each study, the rate of the reaction, ie, $\tan \theta$ or 'slope' (see Fig. 1) and the amount of fibrin'digested in three hours (hereinafter referred to as the three-hour value) were calculated. These measurements were carried out on some 100 blood samples, obtained largely as part of another study, from patients in the postoperative period. In a few samples total lysis had occurred at three hours: these were excluded from the study leaving 92 samples for analysis.

Received for publication 30 September 1971.

\section{Results}

In the 92 studies both the three-hour value and the reaction velocity (slope) were measured. Figure 2 shows the correlation obtained $(r=0.9116)$. Samples in which total lysis of fibrin had occurred by three hours were excluded since the three-hour measurement would underestimate the real activity.

The mean percentage error from the mean in the 92 triplicate readings was $10 \cdot 6 \%$.

\section{Discussion}

Difficulties inherent in the use of lysis time methods when the plasma fibrinogen concentration undergoes alteration have already been discussed (Hickman, 1971). The isotopic method attempts to overcome these difficulties but as put forward originally is time consuming and demanding technically. Furthermore, unless the individual study of fibrin digestion is characterized sufficiently, involving the preparation of numerous aliquots, there is difficulty in determining accurately the rate of the reaction. Thus the assessment of activity by measuring fibrin digestion per unit time is an attractive alternative with advantages both on grounds of accuracy and of technical simplicity.

This study has shown that the three-hour measurement correlates satisfactorily with the overall rate in 92 studies where total lysis of fibrin occurred in not less than three hours. In those samples in which total lysis of fibrin occurs within the three-hour period, the measurements will reflect the total 


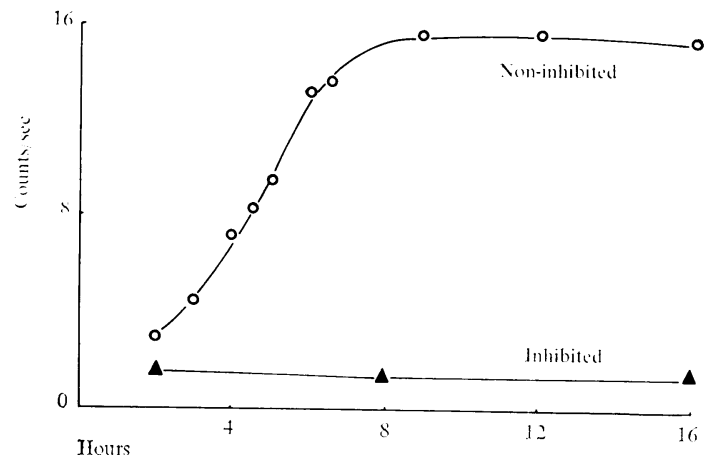

A

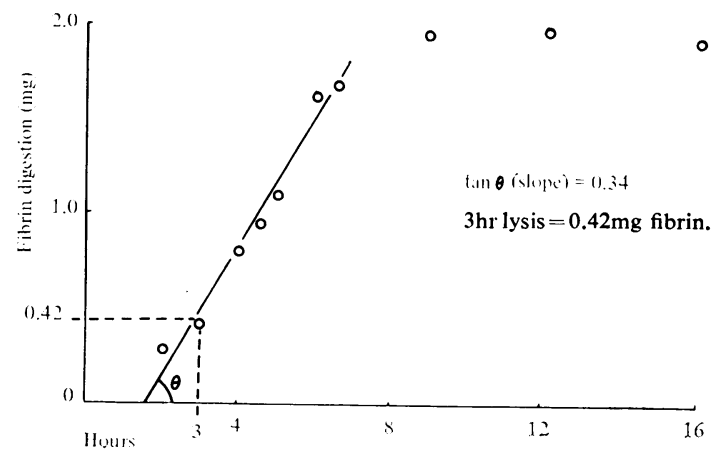

B

Fig. 1A and 1B Illustration of the principle of the isotopic method of measuring fibrinolysis.

A series of aliquots of the blood buffer mixture is incubated at $37^{\circ} \mathrm{C}$. At fixed intervals of time the radioactivity released into the supernatant is measured in individual aliquots and compared with the radioactivity released in aliquots inhibited with EACA. A curve illustrating the reaction is thus obtained (Fig. 1A). Having calculated the specific activity of ${ }^{125}$-I labelled fibrinogen in the blood sample, and after subtraction of the radioactivity in the inhibited series, the reaction may be depicted in terms of $\mathrm{mg}$ fibrin digested per unit time (Fig. 1B). The reaction velocity (slope) is obtained by calculating the tangent of the angle subtended by the regression line drawn to the point at which the reaction reaches the plateau occurring after total fibrin digestion. This article deals with the relationship between the amount of fibrin digested at three hours and the reaction velocity.

amount of fibrin present and tend to underestimate the real fibrinolytic activity of the sample. However, excluding samples of abnormally low fibrin content, total lysis within this period occurs only in samples possessing considerable activity. Nonetheless it is felt that the widest application of the technique lies in the accurate identification of reduced fibrinolytic

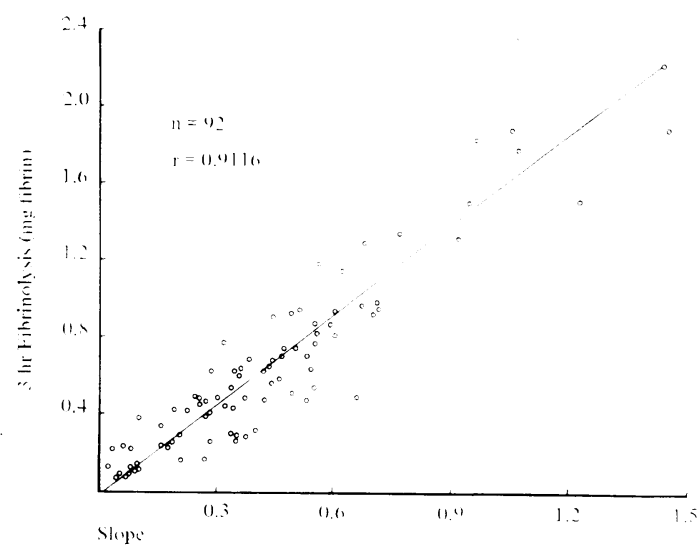

Fig. 2 The three-hour value plotted against the rate of the reaction (slope). The results of 92 studies are plotted and the correlation coefficient $(r)=0.9116$ $(\mathbf{P}<0.001)$.

activity. In view of current interest in the possible association of defective activity with vascular and thrombotic disease it is suggested that there is considerable scope for the useful application of this method. A recommended technique for the measurement of the three-hour value is given in the Appendix.

The authors thank Mr Peter Whitfield and Miss Sylvia Godfrey for technical assistance and Mrs Mary Groves for help with the preparation of the manuscript. They also record with pleasure the interest and helpful criticism of Professor L. P. Le Quesne.

Reference

Hickman, J. A. (1971). A new technique for the quantitative estimation of fibrinolysis using in vivo ${ }^{125}$ I-labelled fibrinogen. Brit. J. Haemat., 20, 611-621.

\section{Appendix}

\section{Recommended Method for Estimation of Three-hour Fibrin Digestion}

Ten $\mathrm{ml}$ of blood is taken by venepuncture using minimal haemostasis. Immediately $4.0 \mathrm{ml}$ is transferred to a conical flask containing $34 \mathrm{ml}$ ice-cold acetate citrate diluent, $p \mathrm{H} 7 \cdot 4$, and surrounded by melting ice. The remaining $6 \mathrm{ml}$ is transferred to a plastic test tube (capacity $10 \mathrm{ml}$ ) containing $40 \mathrm{mg}$ dry EDTA (Stayne Laboratories), and this sample is used subsequently for measuring the plasma fibrinogen concentration and the specific activity of 
the ${ }^{125}$ I-labelled fibrinogen preparation. From the conical flask containing the blood diluent mixture, five $4.75 \mathrm{ml}$ aliquots are transferred by pipette into chilled glass test tubes of internal diameter $15 \mathrm{~mm}$, containing $0.25 \mathrm{ml}$ of freshly prepared thrombin solution strength $50 \mathrm{NIHu} / \mathrm{ml}$ (Leo Pharmaceuticals Ltd, Denmark). To two of these aliquots (hereafter referred to as the inhibited samples) is added $0.1 \mathrm{ml}$ IM EACA solution (13.12 g EACA dissolved in $100 \mathrm{ml}$ of the acetate citrate diluent). Each sample is mixed carefully and placed in a rack in a constant temperature waterbath at $37 \mathrm{C}^{\circ}$. After about 15 minutes each tube is rotated between the palms of the hands to detach the clot from the side of the test tube and allow clot retraction to proceed. Thereafter the tubes are untouched until completion of the test. After three hours' incubation the samples are removed from the waterbath and in the three noninhibited ('test') samples further fibrinolysis is prevented by the addition to each tube of $0.1 \mathrm{ml}$ IM EACA solution. The five test tubes are then placed in a centrifuge at $500 \mathrm{~g}$ for 10 minutes. After centrifugation the clear supernatant is removed with a
Pasteur pipette and filtered by passage through a Pasteur pipette containing a tightly packed plug of cotton wool. Of this filtered supernatant, $3.0 \mathrm{ml}$ is placed in a plastic counting tube, capacity $10 \mathrm{ml}$, and the radioactivity counted in a well-type scintillation counter with a background for ${ }^{125}$ Iodine between 1.0 and 2.0 counts/s.

CALCULATIONS

(1) Radioactivity released by fibrin digestion

(a) Per $3 \mathrm{ml}$ aliquot (Mean count non-inhibited samples - mean count inhibited samples)

(b) Per $0.5 \mathrm{ml}$ whole blood

(Mean count non-inhibited samples - mean

$$
\text { count inhibited samples) } \times \frac{5}{3}
$$

(2) Fibrin digested at three hours ( $\mathrm{mg} / 0.5 \mathrm{ml}$ blood) (Mean count non-inhibited samples - mean count

$$
\text { inhibited samples) } \times \frac{5}{3}
$$

Specific activity of fibrinogen 